\title{
The influence of enhanced UV-B radiation on the spring geophyte Pulmonaria officinalis
}

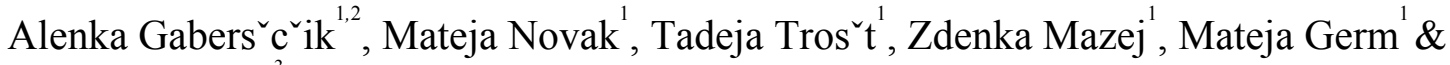 \\ Lars Olof Björn \\ ${ }^{1}$ National Institute of Biology \& ${ }^{2}$ Department of Biology, Biotechnical Faculty, University of Ljubljana, \\ Vecna pot 111, 1001 Ljubljana, Slovenia, ${ }^{3}$ Lund University, Department of Plant Physiology, Box 117, SE- \\ 22100 Lund, Sweden
}

Key words: Photochemical efficiency of PSII, Pigments, Protective compounds, Radiation, Understorey plant, UV-B

\begin{abstract}
Pulmonaria officinalis is an understorey spring geophyte, which starts its vegetative period before full foliation of the tree storey. During its early growth phase it is exposed to full solar radiation, therefore the enhanced UVB radiation could present a threat to this species. An outdoor experiment in which potted plants were exposed to below ambient, ambient, and above ambient (corresponding to 17\% ozone reduction) UV-B radiation, was conducted in order to evaluate the radiation effects. The amount of photosynthetic pigments and photochemical efficiency of PSII were not affected, but the amount of UV-B absorbing compounds was lower in plants grown under reduced UV-B. This change was measurable after only fourteen days in reproductive shoots, while in the vegetative shoots, it was not detectable until after three months. The leaves of $P$. officinalis are variegated and the light green spots became less transparent to PAR under enhanced UV-B. The results reveal that under simulated $17 \%$ ozone depletion the harmful effects of UV-B on the measured parameters were negligible.
\end{abstract}

\section{Introduction}

The thinning of the ozone layer and the concomitant increase in UV-B radiation on the Earth's surface may influence the biota, because of the absorption of UV-B radiation by important biomolecules. Plants respond to UV-B radiation in a species-specific way (Caldwell et al. 1994), balancing the potential dam age and the induction of protective mechanisms. The most frequent response to enhanced UV-B radiation radiation is a production of various secondary substances, primarily UV-B absorbing compounds (Day et al. 1992, 1996), and morphogenetic changes (Greenberg et al. 1996). Changes in the secondary metabolism of plants could alter the interspecific relations among organisms comby influencing the throughflow of energy and cycling of nutrients in the ecosystem.

Until now most investigations have been carried out with higher plants thriving in open places. Pulmonaria officinalis thrives in understoreys of deciduous forests. Its vegetative period starts early in spring and it efficiently uses a brief period before leaves develop on trees, exhibiting a relatively high activity. This time is a temporal window for a UV-B impact because it coincides with the reproduction of $P$. officinalis and plants seem to be more vulnerable to UV-B in the reproductive phase (Tevini \& Teramura 1989). The 
exposure to full sun under the enhanced UV-B could present a threat to this species. An outdoor experiment with supplemental UV-B radiation simulating a $17 \%$ ozone reduction was carried out in order to determine the effects. The changes in photochemical efficiency of PSII as well as the amount of photosynthetic pigments and UV-B absorbing compounds were monitored.

\section{Materials and methods}

\section{Plant material}

Rhizomes of $P$. officinalis were collected in the forest near the city of Ljubljana ( $46^{\circ} 04^{\prime}$ $\left.\mathrm{N}, 14^{\circ} 31^{\prime} \mathrm{E}\right)$ and planted into the pots. In the early vegetative season, i.e., by the end of March, they were exposed to three UV treatments in the Botanical garden in Ljubljana. The experiment lasted three months. During the experiment plants were watered regularly.

\section{$U V-B$ simulation}

A UV-B supplementing system as described by Björn \& Teramura (1993) was used. Three different treatments were applied:

(1) simulation of $17 \%$ ozone depletion (UV-B(+)) using Q-Panel UV-B 313 lamps, filtered with cellulose diacetate filters, which cut out UV-C,

(2) (2) reduced level of UV-B radiation (UV-B(-)) using Mylar foil, which cuts out radiation below about $318 \mathrm{~nm}$ wavelength (Gehrke et al. 1996) and

(3) control: ambient radiation with Q-Panel UV-B 313 lamps filtered with Mylar foil, to include the effects of the UV-A radiation.

The lamps were timer controlled. The times for simulating $17 \%$ ozone depletion were calculated and adjusted weekly using the programs made by Björn \& Murphy (1985), taking into account the generalized plant action spectrum of Caldwell (1968). UV-B biologically effective doses (UV-BBE) are presented in Figure 1. Ambient UV-B radiation was measured using the European Light Dosimeter Network (ELDONET) measuring system which also monitors UV-A radiation and PAR. The data obtained from the system represent unweighted UV-B, which was then converted to plant weighted UV-B using a modification of the program of Björn \& Murphy (1985).

\section{Chlorophyll fluorescence measurements}

The potential and actual photochemical efficiencies of PSII were measured using fluorometer OS-500 (Opti-Science, USA). The potential or maximal photochemical efficiency of PSII was evaluated as $F_{v} / F_{m}$ ratio. Before the measurements, plants were kept in cuvettes in the dark for 15 minutes at ambient temperatures. Photochemical efficiency of PSII under actual light conditions is the photosynthetic quantum yield $(\Phi)$, which is described by the equation $\Phi=\left(F_{m}{ }_{m}-F\right) / F^{\prime}{ }_{m}$. In our experiment yield was measured under full light conditions (from 1500 to $2000 \mu \mathrm{mol} \mathrm{m} \mathrm{s}$ ) and under prevailing ambient temperatures. The measurements were carried out on 6 randomly chosen plants in the third week and after three months. 


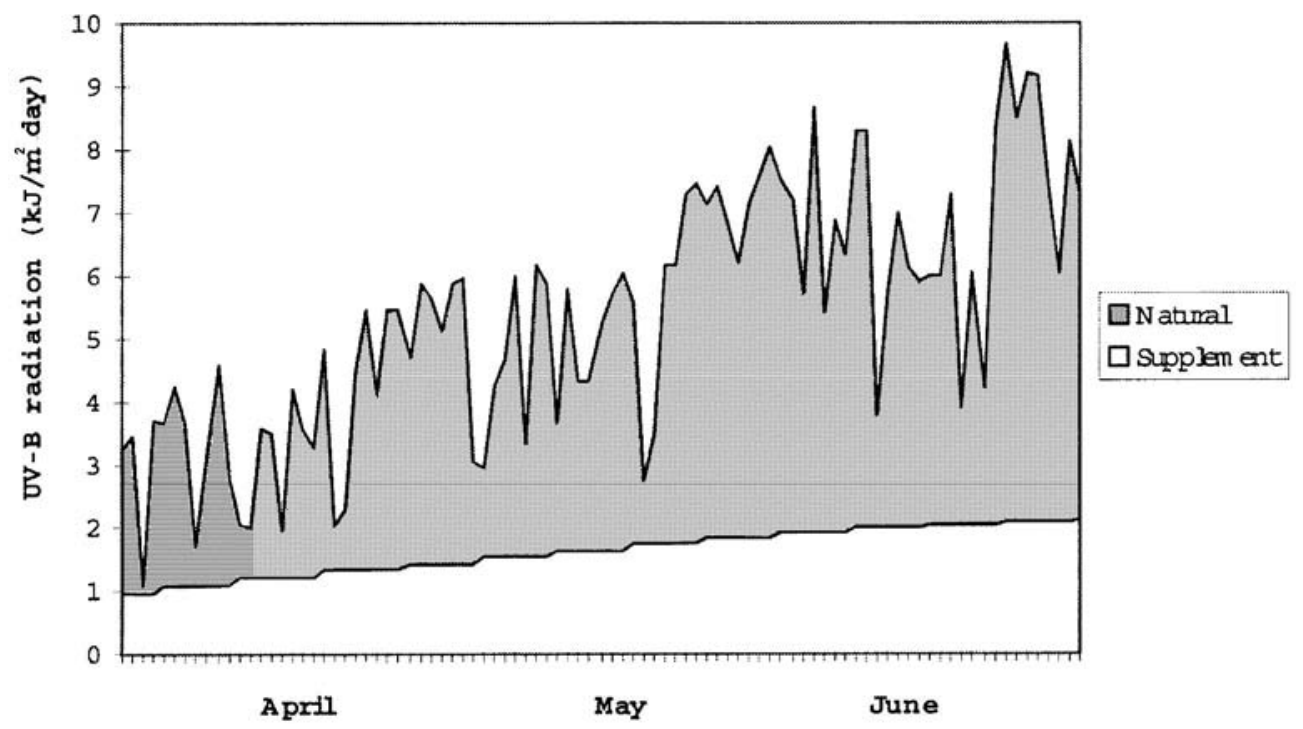

Figure 1. Daily UV-BBE radiation doses for P. officinalis during treatment (supplement - UV-BBE radiation per day corresponding $17 \%$ of ozone depletion, natural - daily measurements by ELDONET instrument converted into UV-BBE).

Photosynthetic pigments and $U V-B$ absorbing compounds

Carotenoids and chlorophylls $a$ and $b$ were determined at different stages of development following the procedure described by Jeffery \& Humphrey (1975). The basic procedure for UV-B absorbing compounds followed the method described by Caldwell (1968). UV$\mathrm{B}$ absorbing compounds were extracted from fresh homogenised plant material with methanol:distilled water: $\mathrm{HCl}=79: 20: 1$. Crude extracts were centrifuged and the absorbance of supernatants was measured in the range from $280-320 \mathrm{~nm}$. The absorbance values were integrated and corrected for the weight of the sample (about $0.1 \mathrm{~g} \mathrm{DW}$ ).

\section{Leaf transmittance}

The penetration of PAR through leaves was measured in dark green parts and in light green spots using the LI-1000 (LI-COR, USA) quantum sensor.

\section{Statistical analyses}

The significance of the differences among treatments was tested by Student's two-tail $t$ test.

\section{Results}

We followed the changes during two growth phases of $P$. officinalis in the period when plants were exposed to full sun. In the first phase at the beginning of the experiment, plants developed reproductive shoots, and simultaneously vegetative rosettes developed. The results of the measurements of photochemical efficiency and yield are presented in Figure 2. Only a small negative effect on yield was found in vegetative shoots under enhanced UV-B radiation. 


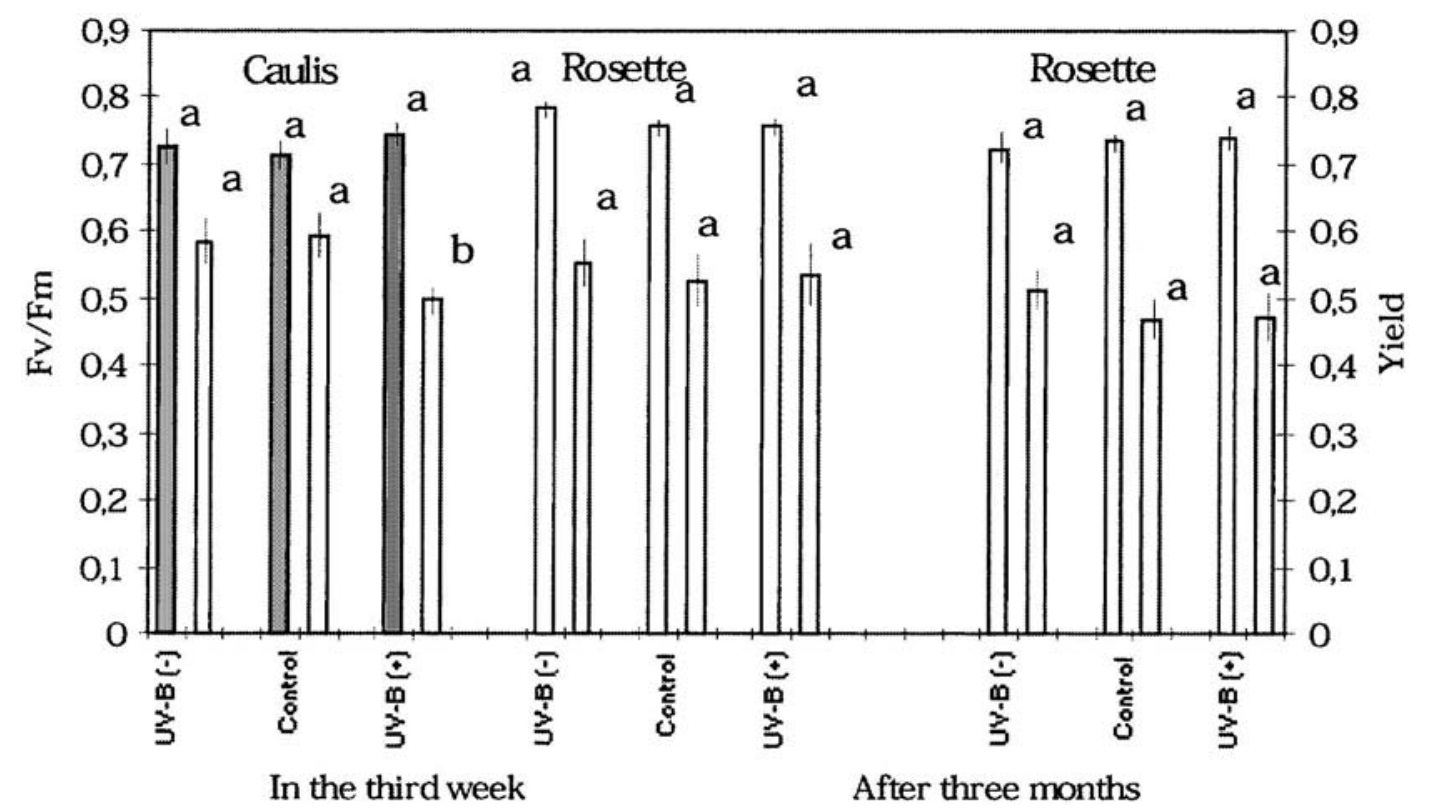

Figure 2. Photochemical efficiency of PSII (grey column) and yield (white column) measured in the leaves of $P$. officinalis in the $3 \mathrm{rd}$ week and after three months of treatment (vertical bars represent the standard error $(n=7-8)$, significance of the differences among treatments, $\leq 0.05)$.

By the end of the experiment, the leaves were examined for morphological features, but there was no evidence of effects of UV-B treatment on trichome density or length, or on specific leaf mass (Table 1). The highest trichome density was found in control plants. The only significant effect of UV-B radiation was an increase in the content UV-B absorbing compounds under control and enhanced UV-B in comparison to reduced UV-B (Figure 3).

Table 1. Some morphological properties of the leaves of $P$. officinalis under different treatments at the end of the experiment (SE: standard error, $n=16)$.

\begin{tabular}{|c|c|c|c|c|c|c|c|}
\hline Treatment & $\begin{array}{l}\text { Leaf } \\
\text { surface }\end{array}$ & $\begin{array}{l}\text { No. of } \\
\text { trichomes } \\
\mathrm{mm}^{-2}\end{array}$ & $\mathrm{SE}$ & $\begin{array}{l}\text { Trichome } \\
\text { length } \\
\mu \mathrm{m}\end{array}$ & $\mathrm{SE}$ & $\begin{array}{l}\text { Specific leaf } \\
\text { weight } \\
\mathrm{g} \mathrm{dm}^{-2}\end{array}$ & $\mathrm{SE}$ \\
\hline \multirow{2}{*}{ UV-B(-) } & Abaxial & 1.68 & 0.11 & 849 & 37 & 0.502 & 0.026 \\
\hline & Adaxial & 1.89 & 0.15 & 615 & 26 & & \\
\hline \multirow[t]{2}{*}{ Control } & Abaxial & 2.39 & 0.11 & 709 & 19 & 0.441 & 0.026 \\
\hline & Adaxial & 2.42 & 0.12 & 573 & 16 & & \\
\hline \multirow[t]{2}{*}{$\mathrm{UV}-\mathrm{B}(+)$} & Abaxial & 1.68 & 0.15 & 719 & 32 & 0.465 & 0.040 \\
\hline & Adaxial & 1.47 & 0.12 & 555 & 14 & & \\
\hline
\end{tabular}

The leaves of $P$. officinalis like many understorey plants are variegated. The dark green lamina is spotted with light green spots. Chlorophyll $a$ and $b$, carotenoids and UV-B absorbing compounds were very similar in dark and light green areas, even though the total amount of chlorophyll was somewhat lower in light green spots (Figure 4). Figure 5 reveals no differences between the dark and light areas with respect to UV-B absorbing pigments. The comparison of different treatments revealed slight but nonsignificant 
differences. In spite of that, the light green spots became less transparent for PAR under enhanced UV-B conditions (Figure 6).

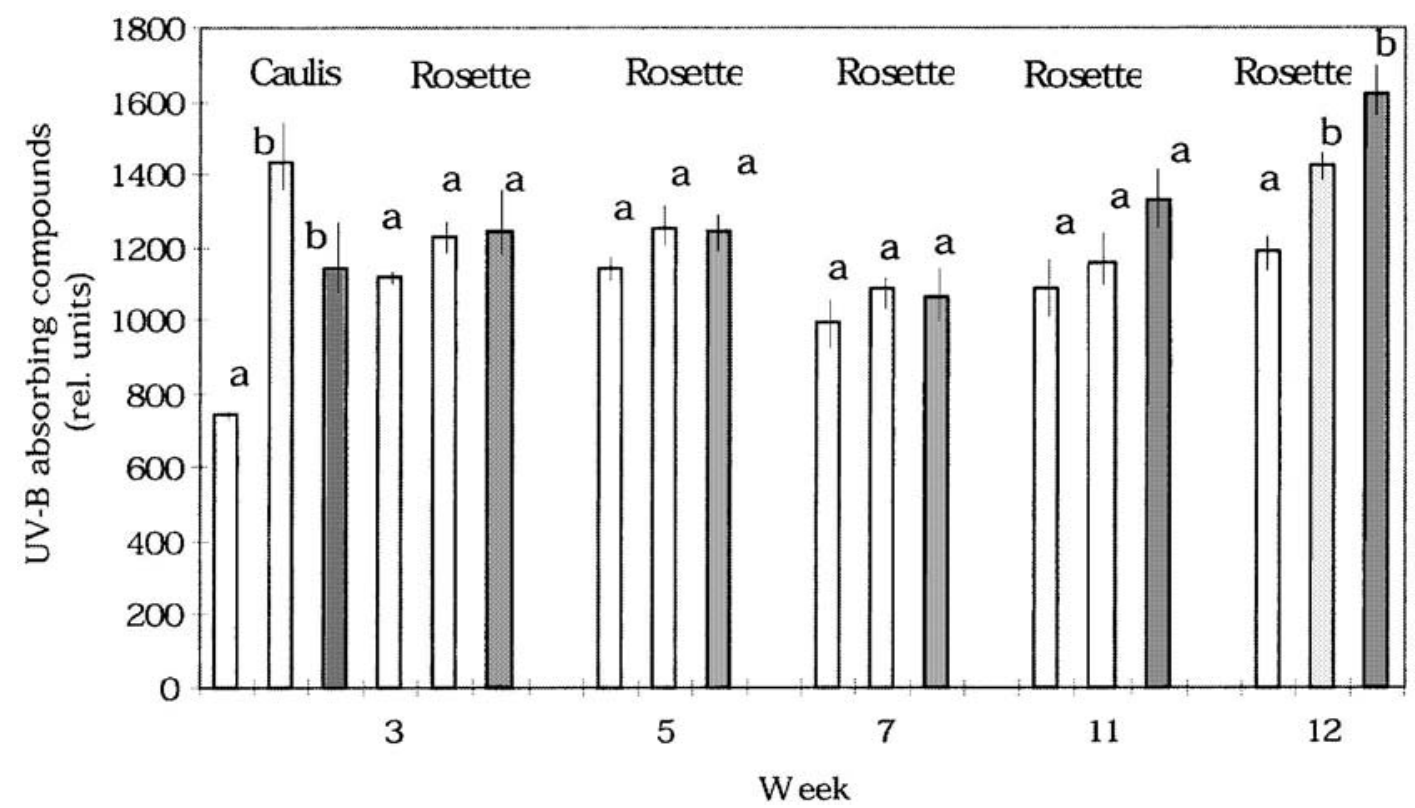

Figure 3. UV-B absorbing compounds in the leaves of $P$. officinalis during the treatment from the beginning of April to the end of June (white - UV-B (-), light gray - control, dark gray - UV-B (+), vertical bars represent the standard error $(n=4)$, significance of the differences among treatments $\leq 0.05)$.

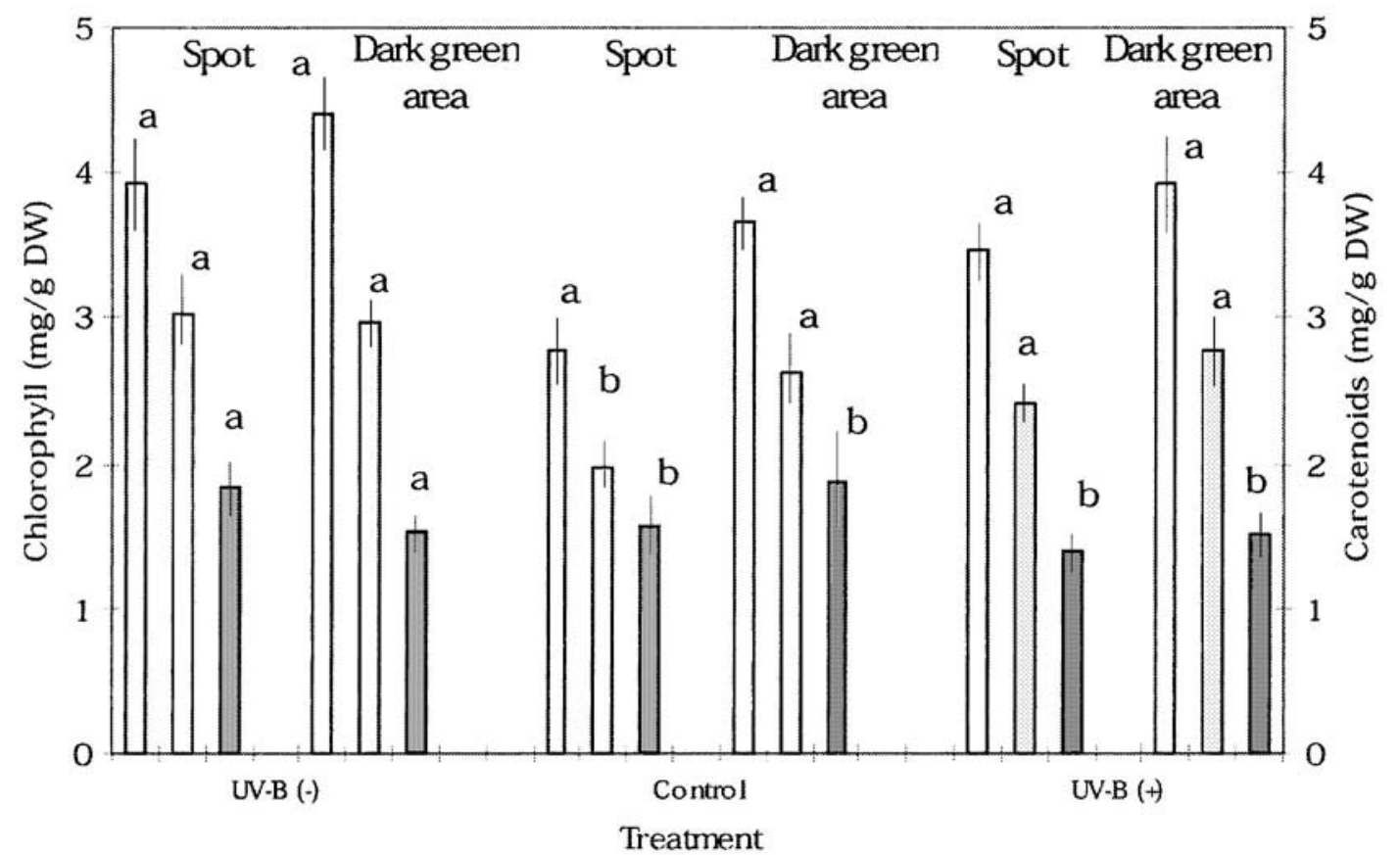

Figure 4. The amounts of chlorophyll $a$ (white) and $b$ (light gray) and carotenoids (dark gray) in light green spots and dark green parts of the leaves of $P$. officinalis at the end of experiment (vertical bars represent the standard error $(n=7-8)$, significance of the differences among treatments, $\leq 0.05)$. 


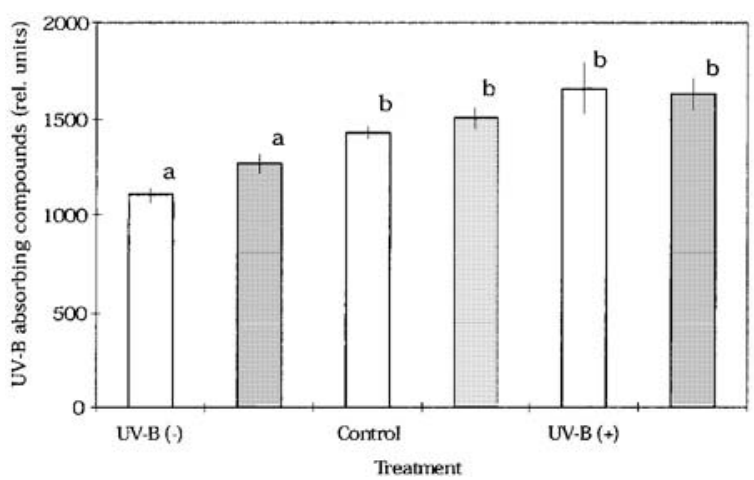

Figure 5. Total amount of UV-B absorbing compounds in light green spots (white) and dark green parts (grey) of the leaves of $P$. officinalis at the end of experiment (vertical bars represent the standard error $(n=$ $8)$, significance of the differences among treatments, $\leq 0.05$ ).

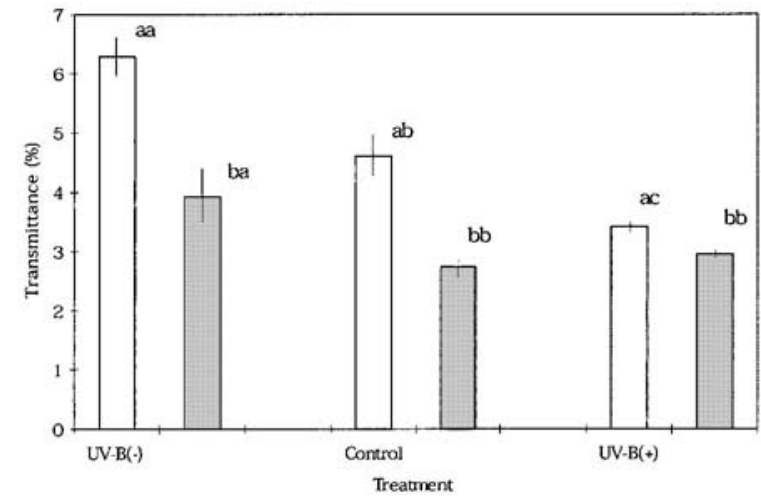

Figure 6. PAR penetration through light green spots (white) and dark green parts (gray) of the leaves of $P$. officinalis after different treatments (vertical bars represent the standard error $(n=8)$, significance of the differences comparing spots and green areas (first sign) and among treatments (second sign), $\leq 0.05$ ).

\section{Discussion}

The growth of $P$. officinalis has two distinct phases. In the first phase, which occurs early in spring when the canopy is still open, plants develop the reproductive shoots. When the reproduction period is concluded, the intensive growth of the vegetative rosette starts, which enlarges the assimilation area (Masarovicova \& Elias 1980). The photosynthetic activity of newly grown leaves during the year supports the reproduction potential in the following year. Increased UV-B therefore influence the success of this species primarily during early spring when the plants are exposed to full solar radiation.

The measurements of photochemical efficiency of PSII do not demonstrate only damage to PSII, but they are also recognised as a powerful way of assessing the effect of various environmental stresses (Ögren \& Öquist 1985). In our experimental plants the $F_{v} / F_{m}$ ratio and yield showed no changes which would indicate harmful influences of UV-B radiation. Veit et al. (1996) report that the synthesis of UV-B absorbing compounds prevents damage to PSII. In $P$. officinalis the relative amounts varied during plant 
development.

A slight, but not significant enhancement in comparison to the ambient UV-B level was observed in June. The comparison with plants grown under a reduced level of UV-B radiation revealed a significant increase. Chlorophyll and carotenoid contents in leaves of $P$. officinalis did not vary significantly among different treatments. It has been reported that in some cases UVB and UV-A radiation can stimulate the chlorophyll and carotenoid synthesis (Rau \& Schrott 1987).

Under elevated UV-B light green spots on leaves became less transparent to PAR, which is probably due to a structural change in the mesophyll, since the pigment content was not affected.

We conclude that on the one hand enhanced UVB radiation corresponding to $17 \%$ ozone depletion affects neither photochemical efficiency of PSII, nor photosynthetic pigments in P. officinalis, even though it is exposed to full solar radiation in the period before the tree foliation. On the other hand the content of UVB absorbing compounds was increased by enhanced UV-B radiation.

\section{Acknowledgements}

This research is part of the project 'The role of UVB radiation on aquatic and terrestrial ecosystems: an experimental and functional analysis of the evolution of protective and adaptive mechanisms in plants', Environment and Climate, PL 970637. The financial support is gratefully acknowledged.

\section{References}

Björn, L. O. \& Murphy, T. M. 1985. Computer calculation of solar ultraviolet radiation at ground level. Physiol. Veg. 23: 555-561.

Björn, L. O. \& Teramura, A. H. 1993. Simulation of daylight Ultraviolet radiation and effects of ozone depletion. Pp. 41-71. In: Young, A. R. (ed.), Environmental UV Photobiology. Plenum Press, New York.

Caldwell, M. M. 1968. Solar ultraviolet radiation as an ecological factor for alpine plants. Ecol.1 Monogr. 38: 243-268.

Caldwell, M. M. \& Flint S. D. 1994. Stratospheric ozone reduction, solar UV-B radiation and terrestrial ecosystems. Climatic Change 28: 375-394.

Day, T. A., Vogelmann, T. C. \& Delucia, E. H. 1992. Are some plant life forms more effective than others in screening out ultra violet -B radiation? Oecologia 92: 513-519.

Day, T. A., Howells, B. W. \& Ruhland, C. T. 1996. Changes in growth and pigment concentrations with leaf age in pea under modulated UV-B radiation field treatments. Plant Cell Environ. 19: 101-108. 
Gehrke, C., Johanson, U., Gwynn-Jones, D., Björn, L. O., Callaghan, T. V. \& Lee, J. A. 1996. Single and interactive effectsof enhanced ultraviolet-B radiation and increased atmospheric CO2 on terrestrial and subarctic ecosystems. Ecol. Bull. 45: 192-203.

Greenberg B. M., Wilson M. I., Gerhardt K. E. \& Wilson K. E. 1996. Morphological and physiological respoonse of Brassica napus to ultraviolet-B radiation: Photomodification of Ribulose-1,5-biphosphate carboxylase/oxydase and potential acclimation processess. J. Plant Physiol. 148: 78-86.

Jeffery, S. W. \& Humphrey, G. F. 1975. New spectrophotometric equations for determining chlorophylls $\mathrm{a}, \mathrm{b}, \mathrm{c}_{1}$ and $\mathrm{c}_{2}$ in higher plants, algae and natural phytoplankton. Biochem. Physiol. Pflanzen 167: 191-194.

Masarovicova, E. \& Elia, P. 1980. Chlorophyll content in leaves of plants in a OakHornbeam Forest. Photosynthetica 14: 580-588.

Ögren, E. \& Öquist G. 1985. Effects of drought on photosynthesis, chlorophyll fluorescence and photoinhibition susceptibility in intact willow leaves. Planta 166: 380388.

Rau, W. \& Schrott, E. L. 1987. Blue light control of pigment biosynthesis. Pp. 43-64. In: Senger, H. (ed.), Blue Light Responses: Phenomena and Occurrence in Plants and Microorganisms 1. CRC Press, Boca Raton, FL.

Tevini, M. \& Teramura, A. H. 1989. UV-B effects on terrestrial plants. Photochem. Photobiol. 50: 479-487.

Veit, M., Bilger, W., Mühlbauer, T., Brummet, W. \& WIinter, K. 1996. Diurnal changes in flavonoids. J. Plant Physiol. 148: 478- 482. 